\title{
Feasting and fasting
}

\author{
Bad nutrition needs the world's attention. Not least that of biologists.
}

1 xterminate the metabolic syndrome!' That was the mission of Japan's government when two vice-ministers for health announced in 2006 that they would cut their waistlines. Encouraged no doubt by the media's keen scrutiny of their progress, they duly did so, with Keizo Takemi's girth coming down from 100.5 to $95 \mathrm{~cm}$ over six months.

As was clear from a wide-ranging survey of nutrition issues at last month's Pacific Health Summit in Seattle, Washington, behavioural change is a key element in tackling the twin crises of under-nutrition and obesity, which are all too easily forgotten in the competition for the world's attention.

What is also insufficiently recognized is the dual nature of the challenge. The combination of "feasting and fasting", as Margaret Chan, director-general of the World Health Organization (WHO), described it in Seattle, increasingly arises as countries develop. For example, China and Mexico are showing both aspects of the 'nutrition transition' - from extreme under-nutrition to obesity caused by insufficient exercise and an excess of unhealthy diets, and diabetes and cancers that follow.

Researchers have tracked the effects of under-nutrition on mothers from before they become pregnant and on their children in the first two years of life. Not only does it cause immediate health problems for the offspring, but it affects their health, educational and work prospects for the rest of their lives. (For an influential overview, see the series published by The Lancet online in January 2008, http://www. thelancet.com/online/focus/undernutrition.) Those longer-term consequences, including an increase in type 2 diabetes, exacerbate the health problems that arise with obesity as countries progress in their development.

Priorities for action to encourage behavioural change include education, compulsory labelling of the calorie content of restaurant menus and regulation of the food industry. Change is also required in farming and retailing practices for healthier food, in both developed and developing countries.

But science matters too. And, above all, science that spans the full range - the study of diets and exercise habits of populations, of physiology, and of molecular mechanisms and genetics - and that includes the social sciences of behaviour as well as the natural sciences of nutrients. For example, cohort studies that examine the dietary intake of women - so often it is women who play a crucial role in public health and who need empowerment - can be related to physiological studies of the effects of dietary components, which in turn can be related to effects of a particular micronutrient on human or
"A holistic approach to under-nutrition has the potential for an immediate impact on policies." model-organism gene expression. Such a holistic approach has the potential for an immediate impact on policies.

Some funding agencies say that they don't receive strong proposals in nutrition. But researchers reasonably assert that funders have only themselves to blame, having weakened organismal biology in favour of cell and molecular studies for many years. A commendable exception to this is France, which for several decades has built and sustained the science of nutrition in a strategic and multifaceted way.

What can best motivate more researchers to tackle these challenges? For a start, an awareness of the sheer impacts of bad nutrition: $35 \%$ of under-five child deaths and $11 \%$ of the global disease burden are caused by under-nutrition, with poor breastfeeding and deficiencies in zinc and vitamin A being major factors. Meanwhile a poor energy balance in individuals lies at the heart of the epidemic in obesity, which has been estimated by the WHO to give rise to as much as $7 \%$ of global health-care costs in developed countries, including a rising tide of diabetes and cancers.

But ultimately, governments need to take the lead. In April, Japan announced a follow-up to its ministers' personal regime of eating less and moving more with a national programme of health check-ups. Next month it hosts the G8 summit, where the cause of improving nutrition will have its opportunity as the participants focus on the food crisis. Science is a critical element requiring the G8's attention in tackling the huge and global burdens of bad nutrition.

\section{In rude health}

\section{A treasure-trove of data in the UK National Health Service is set to energize biomedical research.}

eaching 60 is often associated with people slowing down and beginning to feel their age. Britons, for instance, are entitled to a free bus pass. But their universal health-care system, the National Health Service (NHS), which celebrates its 60th birthday on 5 July, is broadly considered to be in better shape now than it was at its half-centenary - even though baby boomers are greyer and new medicines are dearer. Scrapping it and switching to a private system would be unthinkable.

NHS-bashing has become a UK national pastime, and shortcomings are inevitable in what is one of the world's five biggest employers. But Britons who use the service regularly judge it more positively than those who mainly talk about it. British newspapers are often rife with reports of bad management, but a recent survey showed $91 \%$ of 17 million hospital inpatients rated their care as good, very good or excellent. And although the NHS sometimes fares badly in surveys that focus on how well specific illnesses are treated, probably the most thorough evaluation of late - a six-country study of equity, efficiency, quality, access, and long and productive patient lives by independent foundation the 